\title{
Prevalência da infecção tuberculosa em universitários da Universidade Estadual de Montes Claros, Minas Gerais
}

\author{
Prevalence of tuberculosis infection in students at \\ the State University in Montes Claros, Minas \\ Gerais, Brazil
}

PALAVRAS-CHAVE

- Tuberculose

- Teste tuberculínico

- Estudantes de enfermagem

- Estudantes de medicina
Magna Adaci de Quadros-Coelho ${ }^{\mathrm{I}}$

Cláudia Rocha Biscotto ${ }^{\mathrm{I}}$ Bruna Roberta Tinois ${ }^{\mathrm{I}}$

Fernanda Chaves Freitas ${ }^{\mathrm{I}}$

Juliano Silva Oliveira ${ }^{\mathrm{I}}$

Maria Fernanda Azevedo ${ }^{\mathrm{I}}$

Hercílio Martelli Júnior ${ }^{\mathrm{I}}$

\section{R E S U M O}

O objetivo deste estudo foi conhecer a taxa de infecção pelo bacilo da Tuberculose entre estudantes dos dois primeiros e dois últimos períodos dos cursos de Medicina e Enfermagem da Universidade Estadual de Montes Claros, além de verificar o conhecimento sobre a transmissão e prevenção da tuberculose ocupacional, bem como a utilização destas medidas preventivas. Realizou-se estudo transversal, em que todos os estudantes dos dois períodos iniciais e dois períodos finais do curso de Medicina e de Enfermagem foram submetidos a um questionário padrão e ao teste tuberculínico (PPD), para determinar a prevalência de infecção por tuberculose. Os resultados mostraram diferença de reação ao teste tuberculínico nos estudantes dos períodos iniciais e finais do curso médico, enquanto nos estudantes de Enfermagem não houve diferença entre os períodos. Ficou evidenciado o limitado conhecimento sobre a transmissão e prevenção da aquisição de tuberculose, o que aumenta os riscos de aquisição do bacilo.

\section{KEY WORDS \\ - Tuberculosis \\ - Tuberculin test \\ - Students, Nursing \\ - Students, Medical}

\section{A B S T R A C T}

The study aimed to identify the tuberculosis infection rate among students from the first and last two years in the Schools of Medicine and Nursing at the State University in Montes Claros, Minas Gerais, Brazil, in addition to verifying their knowledge on transmission and prevention of occupational tuberculosis and the use of the recommended preventive measures. A cross-sectional study was conducted in which all the students from the first and last two years of Medicine and Nursing answered a standard questionnaire and had a tuberculin skin test (TST) to determine the prevalence of tuberculosis infection. The results showed a difference in the TST reactivity rate between the first and final years of medical school, while there was no difference in nursing students. The study revealed the students' limited knowledge concerning tuberculosis transmission and prevention, leaving them at increased risk of tuberculosis infection. 


\section{INTRODUÇÃO}

A aquisição da tuberculose (TB) pelos profissionais de saúde tornou-se tema de grande relevância, principalmente com o ressurgimento da doença em países onde até então havia controle epidemiológico ${ }^{1}$. O número estimado de novos casos de TB no ano de 2006 foi de 9,2 milhões, e se trata atualmente da segunda principal causa de morte provocada por doença infecciosa no mundo (depois de HIV / Aids) 2,3. Entre os fatores associados à expansão da TB, verificam-se a piora das condições socioeconômicas da população, deficiências dos sistemas de saúde e o surgimento da síndrome da imunodeficiência adquirida (Aids) $)^{1,2}$. Segundo a Organização Mundial de Saúde (OMS), o Brasil é o décimo sexto país no ranking mundial com maior incidência de TB, e a Região Sudeste apresenta-se como a área de maior taxa de coinfecção da TB com o HIV 2,4.

O expressivo número de surtos de TB em profissionais da saúde tem sido motivo para questionar a segurança em se trabalhar em instituições que admitem elevada quantidade de pacientes com $\mathrm{TB}^{4,5}$. Isto tem resultado em extensiva e frequente revisão dos programas de controle dos Centros de Controle e Prevenção de Doença (CDC $)^{6}$. O Hospital Universitário Clemente de Faria (HUCF), da Universidade Estadual de Montes Claros, Unimontes, é um serviço administrado exclusivamente com recursos do Sistema Único de Saúde (SUS). Atende à população do norte do Estado de Minas Gerais, Vale do Jequitinhonha e sul da Bahia. O HUCF é referência nessas regiões, principalmente para os casos de acidente provocados por animais peçonhentos, Aids e TB. Realizam-se no HUCF sete mil internações e 120 mil consultas anualmente.

É no HUCF que os estudantes de Medicina (EM) e de Enfermagem (EE) da Unimontes atuam em treinamentos clínicos e atividades práticas durante o curso de graduação, estando expostos a ambiente de elevado risco de contaminação pelo bacilo da TB, onde são internados pacientes com TB ativa. Estudo realizado no Rio de Janeiro mostrou ser elevada a prevalência de infecção latente pelo bacilo da TB nos estudantes dos últimos anos do curso médico, e ela foi relacionada com o risco de transmissão nosocomial ${ }^{7}$. Porém, existem poucos dados na literatura latino-americana a respeito do risco de contágio entre EE e EM, além de não ser rotina nas universidades brasileiras a realização do teste tuberculínico (TT) nos estudantes da área da saúde ${ }^{8}$.

Assim, este estudo foi realizado com o objetivo de se conhecer a prevalência da infecção pelo bacilo da TB entre os estudantes de Medicina e de Enfermagem do HUCF e avaliar o conhecimento desses estudantes sobre a transmissão da TB e as medidas preventivas da aquisição intra-hospitalar da doença. Além disso, este estudo suscita o interesse e a discussão do assunto entre os estudantes da área de saúde da instituição.

\section{METODOLOGIA}

\section{Tipo de estudo e local}

Realizou-se um estudo transversal, de base populacional, em 2005, no HUCF da Unimontes. O hospital oferece 144 leitos destinados ao SUS, sendo centro de referência para tratamento de doenças parasitárias e infecciosas, como Aids e TB. A incidência anual de casos de TB no hospital é variável, sendo que no ano de 2004 houve 108 internações, seis óbitos e média de permanência de 14 dias. São oferecidos cinco leitos exclusivamente para o tratamento da TB.

\section{População}

Participaram do presente estudo todos os estudantes do primeiro, segundo e dois últimos períodos dos cursos de graduação em Enfermagem e Medicina, totalizando uma população de 148 universitários. Com duração mínima de quatro anos, ingressam cerca de 30 novos estudantes no curso de Enfermagem da Unimontes a cada semestre. Os graduandos iniciam o contato com pacientes a partir do segundo período. No curso de Medicina, com duração de seis anos, ingressam 30 alunos semestralmente, e desde 2002, com a implantação da metodologia PBL (aprendizagem baseada em problemas), os universitários iniciam o contato com pacientes desde o primeiro período.

\section{Coleta de dados e análise}

Após seleção dos estudantes e treinamento prévio da equipe, aplicou-se questionário estruturado baseado no Risk Assessment Questionnaire $(R A Q)^{9}$. Este instrumento aplicado aos estudantes abordou as seguintes dimensões: idade, vacinação BCG, presença de cicatriz BCG, realização prévia do TT e resultados, contatos anteriores com portadores de $\mathrm{TB}$, episódios pregressos de $\mathrm{TB}$, conhecimentos sobre transmissão e prevenção de TB, utilização de medidas preventivas durante o contato com pacientes em ambiente hospitalar e presença de sinais ou sintomas relacionados à TB. Após conclusão dos questionários, aplicou-se nos estudantes o TT com PPD Rt-23 (Derivado Proteico Purificado), utilizando-se a técnica de Mantoux, com treinamento prévio por intermédio do material didático cedido pelo Center for Disease Control (CDC). Após 48 a 72 horas da administração do teste, os participantes retornaram para avaliação e medida da enduração. A reação foi avaliada por especialistas (Magna Adaci de Quadros Coelho e Cláudia Rocha Biscotto), que utilizaram o método da visualização, palpação, delineamento do contorno com caneta esferográfica e medida do diâmetro com régua milimetrada, padronizada pelo CDC.

Em seguida, inferiu-se que os casos não reatores $(0 \mathrm{~mm}$ a 4 $\mathrm{mm}$ ) representaram indivíduos não reatores ou anérgicos e foram submetidos a novos testes no mínimo duas semanas após, 
para confirmação do resultado. O reator fraco (5 $\mathrm{mm}$ a $9 \mathrm{~mm})$ indicou indivíduo infectado pelo bacilo ou por outras micobactérias, e o reator forte (10 mm ou mais) apontou infecção pelo Mycobacterium tuberculosis, assintomático ou não. No estudo, estes indivíduos foram encaminhados ao especialista para realização de exame clínico e de propedêutica adequada. Os estudantes não reatores prosseguiram prospectivamente no teste de hipótese sobre transmissão nosocomial de TB, na própria instituição.

Este estudo foi realizado com aprovação do Comitê de Ética em Pesquisa (112/2004) envolvendo Seres Humanos da Unimontes, e os respondentes assinaram termo de consentimento informado livre e esclarecido, de acordo com a Resolução 196/96 do Conselho Nacional de Saúde.

\section{RESULTADOS}

Foram avaliados 148 universitários de Enfermagem e Medicina da Unimontes, no primeiro semestre de 2005, sendo 44 estudantes dos períodos iniciais e 27 dos períodos finais do curso de Medicina e 41 estudantes dos períodos iniciais e 36 dos períodos finais do curso de Enfermagem. Na análise da reação ao TT, observou-se que $18,2 \%$ e $37 \%$ dos estudantes dos períodos iniciais e finais, respectivamente, do curso médico foram fortemente reatores ao PPD (RP = 2,65; IC 95\% 0,89-7,91; p = 0,076) (Tabela 1). Entre os estudantes de Enfermagem, não houve diferença da reação ao teste tuberculínico entre os períodos iniciais e finais (RP = 0,72; IC 95\% 0,26-1,95; p =0,51) (Tabela 1).

Em relação ao conhecimento dos meios de transmissão da TB, 47,7\% dos estudantes dos períodos iniciais de Medicina e

Tabela 1

Respostas sobre a leitura do PPD nos estudantes dos períodos iniciais e finais dos cursos de Medicina e Enfermagem

\begin{tabular}{|c|c|c|c|c|c|}
\hline & & \multicolumn{3}{|c|}{ Leitura PPD } & \\
\hline \multicolumn{2}{|c|}{ Período e Curso } & \multirow{2}{*}{$\frac{\text { Não/Fraco }}{n}$} & \multirow{2}{*}{$\frac{\text { Forte }}{36}$} & \multicolumn{2}{|c|}{ Total } \\
\hline $1^{\circ}$ e $2^{\circ}$ & Medicina* & & & 8 & 44 \\
\hline & & $\%$ & 81,8 & 18,2 & 100 \\
\hline \multirow[t]{2}{*}{$11^{\circ}$ e $12^{\circ}$} & Medicina* & $\mathrm{n}$ & 17 & 10 & 27 \\
\hline & & $\%$ & 63 & 37 & 100 \\
\hline \multirow[t]{2}{*}{ Total } & & $\mathrm{n}$ & 53 & 18 & 71 \\
\hline & & $\%$ & 74,6 & 25,4 & 100 \\
\hline \multirow[t]{2}{*}{$1^{\circ}$ e $2^{\circ}$} & Enfermagem $* *$ & $\mathrm{n}$ & 28 & 13 & 41 \\
\hline & & $\%$ & 68,3 & 31,7 & 100 \\
\hline \multirow[t]{2}{*}{$7^{\circ}$ e $8^{\circ}$} & Enfermagem ${ }^{* *}$ & $\mathrm{n}$ & 27 & 9 & 36 \\
\hline & & $\%$ & 75 & 25 & 100 \\
\hline \multirow[t]{2}{*}{ Total } & & $\mathrm{n}$ & 55 & 22 & 77 \\
\hline & & $\%$ & 71,4 & 28,6 & 100 \\
\hline
\end{tabular}


$26,8 \%$ dos estudantes dos períodos iniciais de Enfermagem referiam desconhecê-los, sendo este dado estatisticamente significativo ( $R P=0,40 ;$ IC 95\% 0,16-0,99; p = 0,047) (Tabela 2). Resultado semelhante foi observado quando se avaliou o conhecimento sobre as medidas de prevenção a TB, com $88,6 \%$ dos estudantes dos períodos iniciais do curso médico referindo desconhecimento, comparados com $56,1 \%$ dos estudantes dos períodos iniciais do curso de Enfermagem ( $\mathrm{RP}=0,16$; IC 95\% 0,05-0,50; p = 0,001) (Tabela 2). Quando avaliado o uso de máscaras com filtro ao entrar em contato com pacientes com TB ativa, verificou-se maior aderência à medida entre os estudantes do último ano de Enfermagem, quando comparado aos estudantes dos últimos períodos de Medicina, sendo esta diferença estatisticamente significativa ( $R P=0,13$; IC 95\% 0,04-0,46; $p=0,02$ ) (Tabela 3).

\section{DISCUSSÃO}

Em países onde houve o ressurgimento da TB e o aparecimento de altos índices de resistência a múltiplas drogas pelo bacilo, o risco de TB infecção e TB doença aumentou significativamente entre os profissionais de saúde ${ }^{10}$. Em países em desenvolvimento, como o Brasil, há escassez de estudos a respeito do risco de TB entre estudantes de Medicina. Apesar das diretrizes da OMS sobre a TB nas instituições de saúde, não existe nenhum guia a respeito das atividades a serem implementadas em cursos de graduação na área da saúde ${ }^{7}$.

O presente estudo evidenciou maior índice de infecção tuberculosa entre os EM do último ano quando comparado aos estudantes do primeiro ano, embora estatisticamente não significativo $(p=0,076)$. Em estudo realizado na Faculdade de Medici-

Tabela 2

Respostas sobre o conhecimento a respeito das formas de transmissão e medidas preventivas da TB de acordo com cursos de graduação (Medicina e Enfermagem) nos primeiros períodos

\begin{tabular}{|c|c|c|c|c|c|}
\hline \multirow[b]{2}{*}{ Período e Curso } & & \multicolumn{4}{|c|}{ Conhecimento sobre meios de transmissão } \\
\hline & & Sim & Não & & \\
\hline \multirow[t]{2}{*}{$1^{\circ}$ e $2^{\circ}$} & Medicina* & $\mathrm{n}$ & 23 & 21 & 44 \\
\hline & & $\%$ & 52,3 & 47,7 & 100 \\
\hline \multirow[t]{2}{*}{$1^{\circ}$ e $2^{\circ}$} & Enfermagem* & $\mathrm{n}$ & 30 & 11 & 41 \\
\hline & & $\%$ & 73,2 & 26,8 & 100 \\
\hline \multirow[t]{3}{*}{ Total } & & $\mathrm{n}$ & 53 & 32 & 85 \\
\hline & & $\%$ & 62,4 & 37,6 & 100 \\
\hline & & & \multicolumn{3}{|c|}{ Conhecimento sobre medidas preventivas } \\
\hline \multicolumn{2}{|l|}{ Período e Curso } & Sim & Não & \multicolumn{2}{|c|}{ Total } \\
\hline \multirow[t]{2}{*}{$1^{\circ}$ e $2^{\circ}$} & Medicina** $^{* *}$ & $\mathrm{n}$ & 5 & 39 & 44 \\
\hline & & $\%$ & 11,4 & 88,6 & 100 \\
\hline \multirow[t]{2}{*}{$1^{\circ}$ e $2^{\circ}$} & Enfermagem $* *$ & $\mathrm{n}$ & 18 & 23 & 41 \\
\hline & & $\%$ & 43,9 & 56,1 & 100 \\
\hline \multirow[t]{2}{*}{ Total } & & $\mathrm{n}$ & 23 & 62 & 85 \\
\hline & & $\%$ & 27,1 & 72,9 & 100 \\
\hline
\end{tabular}

\footnotetext{
${ }^{*} \mathrm{p}=0,047$

** $\mathrm{p}=0,001$
} 
Tabela 3

Respostas sobre a utilização de máscara com filtro por estudantes de graduação (Medicina e Enfermagem) nos últimos períodos dos cursos de graduação

\begin{tabular}{cccccc}
\hline & & \multicolumn{3}{c}{ Utilização de máscara com filtro } & \\
\multicolumn{2}{c}{ Período e Curso } & Sim & Não & Total \\
\hline \multirow{2}{*}{$11^{\circ}$ e $12^{\circ}$} & Medicina & $\mathrm{n}$ & 4 & 23 & 29 \\
& & $\%$ & 14,8 & 85,2 & 100 \\
\multirow{2}{*}{$7^{\circ}$ e $8^{\circ}$} & Enfermagem & $\mathrm{n}$ & 20 & 15 & 35 \\
& & $\%$ & 57,1 & 42,9 & 100 \\
\hline \multirow{2}{*}{ Total } & $\mathrm{n}$ & 24 & 38 & 62 \\
& & $\%$ & 38,7 & 61,3 & 100 \\
\hline
\end{tabular}

$* \mathrm{p}=0,02$

na e Ciências da Saúde da UFRJ, foi sugerido que estudantes de Medicina apresentam risco aumentado de aquisição de TB em ambiente hospitalar ${ }^{8}$. Em outro estudo na mesma instituição (UFRJ), os EM foram comparados aos estudantes de Engenharia Química e apresentaram maior prevalência de positividade ao teste tuberculínico, especialmente na medida em que avançavam no curso $(4,6 \%$ e $16,2 \%$ nos anos iniciais e finais do curso, respectivamente, $p<0,001$ ), período este que os estudantes passam a ter maior contato com pacientes ${ }^{11}$. Esses resultados diferem dos índices encontrados no presente estudo, onde $18,2 \%$ e $37 \%$ dos estudantes dos primeiros e dos últimos períodos de Medicina foram, respectivamente, fortes reatores ao teste tuberculínico. Essa diferença poderia ser explicada pelo número reduzido da população ou ainda pelo alto índice de exposição ao bacilo apresentado pelos estudantes da Unimontes, cujo hospital e ambulatórios referenciam pacientes com TB e HIV, de toda a região norte do Estado de Minas Gerais e também do sul do Estado da Bahia.

Os EM da Unimontes se inserem na prática clínica a partir do primeiro período da graduação no HUCF. Entretanto, verifica-se relevante escassez de conhecimentos sobre as formas de transmissão e de prevenção da aquisição ocupacional de TB, o que aumenta substancialmente os riscos de aquisição do bacilo, já que o conhecimento individual sobre os meios de transmissão e prevenção da TB constitui um dos principais fatores que contribuem para os altos índices de TB entre profissionais de saúde ${ }^{12}$. Recentemente, Trajman et al..$^{13}$ avaliaram a abordagem do ensino referente à TB em cursos médicos brasileiros, envolvendo as diversas regiões geográficas do País e instituições públicas e privadas. Observaram que as instituições dedicam em seus projetos político-pedagógicos uma média de 27 horas ao estudo da ET, distribuídas em diversas disciplinas. Verificaram, ainda, que o teste para HIV para os portadores de TB foi oferecido em somente $54 \%$ dos cursos médicos. Assim, concluem que o ensino pertinente a TB no Brasil se encontra fragmentado e que são necessárias mudanças curriculares.

Quando se comparam os EM com os colegas do curso de Enfermagem, verifica-se menor nível de conhecimento tanto sobre as formas de transmissão e prevenção da aquisição ocupacional da TB, como da utilização de máscaras com filtro. Os EE não se inserem tão precocemente na prática hospitalar como os EM. É provável que a ausência de medidas administrativas e a utilização inadequada de medidas de proteção respiratória individual sejam as principais razões para o maior risco de aquisição da TB pelos profissionais de saúde em relação à população geral $^{12}$.

A possibilidade de se adquirir TB não pode ser totalmente eliminada, mas um importante ponto de partida para o controle da TB infecção é a orientação sobre a transmissão e o conhecimento das medidas de prevenção. Estas orientações incluem: controles administrativos (avaliação do risco de infecção, formação eficiente dos profissionais de saúde, diagnóstico precoce, educação do paciente, triagem correta, avaliação do programa de controle de infecção hospitalar), controles ambientais (au- 
mentar a ventilação natural, ventilação mecânica, radiação ultravioleta, germicida, luz e limpeza dos aparelhos de ar-condicionado) e proteção respiratória pessoal (uso de máscaras com filtro $)^{14}$. Estas orientações também devem ser dirigidas de maneira especial aos novos profissionais de saúde. A proteção dos profissionais começa antes da admissão e se mantém durante a sua atuação. Isto inclui a adoção de medidas de segurança durante o cuidado com os pacientes e medidas de detecção precoce da infecção tuberculosa pelos profissionais de saúde.

Com a amplitude de materiais em todas as áreas de conhecimento sobre a TB, é desconcertante que médicos continuem a cometer erros frequentes no diagnóstico e tratamento da $\mathrm{TB}^{15}$. É cada vez mais comum a atuação de equipes multiprofissionais de saúde na área de cuidados intensivos, mas, apesar das funções partilhadas, esses profissionais recebem formações muito distintas ${ }^{15}$. Nos Estados Unidos, existe um consórcio curricular de escolas da área da saúde com o objetivo de instigar conhecimentos, habilidades e atitudes adequadas no manejo e controle de TB ativa e latente entre estudantes da graduação, além de estimular a educação continuada ao longo da carreira ${ }^{16}$. Portanto, há necessidade de intensificar a educação dos trabalhadores da saúde e dos futuros profissionais sobre a TB infecção e seu controle.

Não existe recomendação a respeito da testagem tuberculínica anual de estudantes de Medicina no Brasil ${ }^{8}$. As diretrizes do CDC recomendam que todos os profissionais de saúde susceptíveis (potencialmente expostos a pacientes com TB ativa) sejam submetidos ao TT pelo menos uma vez ao ano ${ }^{17}$. Um programa de testagem cutânea rotineira e diretrizes específicas de controle da TB infecção são necessários para os estudantes da área da saúde. Outros estudos realizados no Brasil também sugerem que os estudantes de Medicina e Enfermagem apresentam maior risco para infecção nosocomial pelo Mycobacterium tuberculosis e recomendam a criação de um programa contra a TB nas faculdades de saúde, especialmente nas cidades com maior ocorrência de $\mathrm{TB}^{7,18}$.

\section{CONCLUSÃO}

O presente estudo possibilitou ampliar a reflexão/discussão sobre as questões de segurança dos estudantes e dos profissionais de saúde que atuam no HUCF da Unimontes. Este estudo mostrou a vulnerabilidade desta população em relação à TB e revelou a necessidade de implementar medidas específicas de prevenção para os estudantes da área de saúde, além de alerta para o uso de medidas de controle ambiental, incluindo isolamento respiratório de casos confirmados ou suspeitos de TB e a prote- ção respiratória individual durante o atendimento ao paciente infectado. É necessário um programa de testagem tuberculínica anual em estudantes e nos outros profissionais da saúde, para identificar novas infecções.

\section{AGRADECIMENTOS}

A todos os estudantes dos cursos de Medicina e Enfermagem que participaram deste estudo; à professora Rita de Cássia Filocre Saraiva, coordenadora de Imunização do DRS, Montes Claros (MG); ao Conselho Nacional de Desenvolvimento Científico e Tecnológico (CNPq) (HMJ).

\section{REFERÊNCIAS}

1. Joshi R, Reingold AL, Menzies D, Pai M. Tuberculosis among health-care workers in low and middle-income countries: a systematic review. PLoS Med. 2006;3:494.

2. World Health Organization. Global tuberculosis control [on line]. [acesso em 22 amio 2008]. [s.1.]: WHO report; 2008. Disponível em: http:/ / www.who.int/tb/publications/global_report/2008/pdf/fullreport.pdf .

3. Young DB, Perkins MD, Duncan K, Barry CE. Confronting the scientific obstacles to global control of tuberculosis. J Clin Invest. 2008;118(2):1255-1265.

4. Brasil. Ministério da Saúde. Secretaria de Vigilância em Saúde. Guia de Vigilância Epidemiológica. $6^{\circ}$ ed. Brasília: Ministério da Saúde; 2005. p. 732.

5. Barsegian V, Mathias KD, Wrighton-Smith P, Grosse-Wild $\mathrm{H}$, Lindermann MP. Prevalence of latent tuberculosis infection in German radiologists. J Hosp Infect. 2008;69(4):69-76.

6. CDC-Centers for Disease Control and Prevention, Trends in tuberculosis - United States, 2007. MMWR Morbidity mortality Whly Rep. 2008;21(6):281-285.

7. Teixeira EG, Menzies D, Comstock GW, Cunha AJ, Kristki AL, Soares LC. Latent tuberculosis infection among undergraduate medical students in Rio de Janeiro State, Brazil. Int J Tuberc Lung Dis. 2005;9(5):841-847.

8. Silva VMC, Cunha AJLA, Kritski AL. Tuberculin skin test conversion among medical students at a teaching hospital in Rio de Janeiro, Brazil. Infect Control Hosp Epidemiol. 2002;23(1):591-594.

9. Koppaka VR, Harvey E, Mertz B, Johnson BA. Risk assessment in targeted TB screening. Clin Infect Dis. 2003;36(2):599-607. 
10. Ratio M, Helenius H, Tala E. Is the risk of occupational tuberculosis higher for young health care workers? Int J Tuberc Lung Dis. 2003;7(2):556-562.

11. Silva VM, Cunha AJ, Oliveira JR, Figueira MM, Nunes ZB. Medical students at risk of nosocomial transmission of Mycobacterium tuberculosis. Int J Tuberc Lung Dis. 2000;4(3):420-426.

12. Humphreys H. Control and prevention of healthcare-associated tuberculosis: the role of respiratory isolation and personal respiratory protection. J Hosp Infect. 2007;66(2):1-5.

13. Paul A, Jensen PA. Where should infection control programs for tuberculosis begins? Int J Tuberc Lung Dis. 2005;9(3):825-829.

14. Trajman A, Correia N, Venturi M, Kritski A, Rufino-Neto A, Scatena Villa TC. Tuberculosis teaching in Brazilian medical scholls. Int J Tuberc Lung Dis. 2007;11(6):703-705.

15. Jackson M, Harrity S, Hoffman H, Catanzaro A. A survey of health professions students for knowledge, attitudes and confidence about tuberculosis, 2005. BMC Public Health. 2007;7(1):219-223.

16. Harrity S, Jackson M, Hoffman H, Catanzaro A. The National Tuberculosis Curriculum Consortium: a model of multi-disciplinary educational collaboration. Int J Tuberc Lung Dis. 2007;11(2):270-274.

17. Nicas M. Regulating the risk of tuberculosis transmission among health care workers. Am Ind Hyg Assoc J. 2000;61(5):334-339.
18. Maciel EL, Meireles W, Silva AP, Fiorotti K, Dietze R. Transmissão nosocomial por Mycobacterium tuberculosis nos estudantes da área de saúde em uma região de alta incidência de tuberculose, Vitória, Estado do Espírito Santo. Rev Soc Bras Med Trop. 2007;40(6):397-399.

\section{CONTRIBUIÇÃO DOS AUTORES}

Magna A. Q. Coelho e Cláudia R. Biscoto participaram da coordenação do estudo, orientação da coleta de dados, execução e leitura dos testes, discussão dos resultados e redação do artigo científico. Bruna Roberta Tinois, Fernanda C. Freitas, Juliano S. Oliveira e Maria Fernanda Azevedo aplicaram os questionários e construíram o banco de dados. Hercílio M. Júnior participou da análise dos resultados, discussão e redação do artigo científico.

\section{CONFLITO DE INTERESSES}

Declarou não haver

\section{ENDEREÇO PARA CORRESPONDÊNCIA}

Hercílio Martelli Júnior

Rua Iracy de Oliveira Novaes, 220 - apto 207A

Cândida Câmara - Montes Claros

CEP. 39401-043 MG

E-mail: hmjunior2000@yahoo.com 